\title{
Effets d'une séquence d'écriture collaborative sur l'apprentissage du lexique au 3e cycle du primaire
}

\author{
Maude Roy-Vallières \\ Université du Québec à Montréal \\ Anila Fejzo \\ Université du Québec à Montréal
}

\begin{abstract}
Résumé
Cette recherche explore les effets d'une séquence d'écriture collaborative sur l'apprentissage du lexique chez des élèves de $6^{\mathrm{e}}$ année du primaire scolarisés en français. Deux groupes de sixième année ont participé à cette étude quasi expérimentale. Chaque groupe a participé à deux séances de préparation, ainsi que trois séances d'écriture précédées par un enseignement explicite de 5 mots cibles. Les participants du groupe expérimental écrivaient en équipe de quatre tandis que ceux du groupe contrôle écrivaient individuellement. Avant et après l'intervention, des mesures de la connaissance des mots ciblés par l'intervention ont été prises auprès des deux groupes de recherche. Les résultats d'une analyse de variance ANOVA n'ont révélé aucune différence significative entre les deux groupes sur le plan de l'apprentissage des mots cibles. Nos résultats suggèrent que l'écriture collaborative et l'écriture individuelle mènent à un apprentissage semblable des mots et invitent à réfléchir aux conditions qui favorisent l'enseignement du lexique.
\end{abstract}

\begin{abstract}
This study examined the effects of a collaborative writing sequence on vocabulary learning among French elementary school students. Two sixth grade groups participated in this quasiexperimental study. Each group completed two preparation sessions and three writing sessions preceded by the explicit teaching of five target words. Participants in the experimental group wrote in teams of four while those in the control group wrote individually. Participants' target word knowledge was measured prior to and upon completion of the intervention. Results of an ANOVA analysis of variance revealed no significant difference between the two groups on target word knowledge following intervention. Our findings suggest that collaborative writing and individual writing both lead to similar acquisition of vocabulary. Strategies for promoting vocabulary acquisition in the classroom are discussed.
\end{abstract}




\section{Effets d'une séquence d'écriture collaborative sur l'apprentissage du lexique au 3e cycle du primaire}

\section{Introduction}

Pour un élève du primaire, le nombre de mots qu'il connaît est relié à sa réussite scolaire (Ardasheva et coll., 2019; Binder et coll., 2017; Gablasova, 2014; Graves, 2006; Guthrie et coll., 2004; Ouellette, 2006; Yonek, 2009; Zhang et Yang, 2016). En effet, la quantité de mots connus peut influer sur la compréhension en lecture et sur la compétence en écriture. La connaissance d'environ 98\% des éléments lexicaux d'un texte serait nécessaire pour une compréhension adéquate et autonome de celui-ci (Zhang et Yang, 2016). Lorsqu'on prend en compte que la majorité des matières abordées à l'école primaire font appel à la lecture de textes, on voit ici toute l'importance de l'étendue lexicale pour la réussite scolaire (Magnusson et coll., 2019; Vinterek et coll., 2018). En outre, il s'avère que le bagage lexical nécessaire pour réussir varie en fonction des matières, constituant un défi supplémentaire pour le lecteur (Beacco et coll., 2016; Kwary, 2011). D’un autre côté, cette étendue joue un rôle important dans l'écriture. Selon le modèle de Hayes et Flowers (1986), la sélection des mots peut se retrouver dans la planification, la mise en texte et la révision d'une situation d'écriture. La connaissance d'un grand nombre de mots et l'établissement de leurs représentations précises dans la mémoire à long terme faciliteraient la transposition des idées en mots et permettraient de libérer des ressources cognitives pour d'autres éléments plus complexes du processus, rendant la tâche d'écriture plus aisée. Le développement du lexique peut ainsi avoir un impact important sur la réussite scolaire des jeunes du primaire qui n'ont pas encore automatisé le processus d'écriture.

Pourtant, on remarque que l'enseignement du lexique est le parent pauvre dans l'apprentissage de la langue (Anctil, 2011; Beck et coll., 2002). Dans une étude de Scott, et coll. (2003), le temps accordé à l'enseignement lexical dans des écoles primaires canadiennes anglophones représentait 1,4\% du temps total passé à l'école. En outre, le lexique était enseigné de façon isolée, sans être rattaché à d'autres matières, le quart du temps, s'opposant aux écrits scientifiques favorisant l'enseignement des mots en contexte (Bolger et coll., 2008; Dreyfus, 2004; Gablasova, 2015; Graves, 2006). Enfin, les enseignants du primaire utiliseraient majoritairement des techniques d'enseignement du lexique qui sont peu appréciées des apprenants (Beck et coll., 2002, Bridgman, 2005, Kamil et Hiebert, 2005), telles que l'apprentissage par cœur de définitions ou l'explication des mots par l'enseignant (Dreyfus, 2004). Or, les dernières années du primaire constitueraient pourtant un moment privilégié pour enseigner le lexique, les jeunes de cet âge ayant accès à plus de ressources cognitives dû à l'automatisation du processus d'écriture (Perez, 2018). En outre, les enseignants du secondaire auraient tendance à peu aborder les connaissances lexicales (Anctil, 2011), rendant une certaine maitrise de cellesci préalable à la réussite à ce cycle d'enseignement.

L'identification des lacunes dans l'enseignement actuel du lexique souligne la nécessité d'explorer davantage les techniques et les outils favorisant son apprentissage, afin d'aider les élèves à bonifier leurs connaissances lexicales. Toutefois, il parait d'abord pertinent de dégager les composantes de la connaissance des mots pour orienter le choix d'un dispositif favorisant leur apprentissage. 


\section{Les aspects de la connaissance des mots}

La connaissance d'un mot est un concept complexe et multidimensionnel, qui ne se limite pas à la maitrise de la forme orale ou écrite du mot. Les chercheurs qui s'intéressent à la connaissance des mots (Beck et coll., 2002; Nagy et Scott, 2000; Nation, 1990) identifient cinq à huit facettes qui, d'une manière générale, regroupent les formes phonologique et orthographique du mot, le ou les différents sens du mot, les propriétés grammaticales et les liens avec les autres mots. De plus, ils soutiennent que l'apprentissage d'un mot est un processus progressif passant d'une connaissance partielle du mot à la maitrise de tous ses aspects. Il s'agit d'un concept commun chez ces chercheurs, désigné sous le terme d'incrémentalité. L'incrémentalité est parfois conçue comme une propriété de la connaissance des mots (Nagy et Scott, 2000). Dans cette même optique, Beck et coll. (2002) distinguent cinq étapes de la connaissance des mots, variant de « connaissance nulle » à « connaissance riche et décontextualisée du mot, de ses relations avec d'autres mots et de ses utilisations métaphoriques » [Traduction libre] (Beck et coll., 2002, p. 11).

Il en résulte que la connaissance parfaite d'un mot est complexe et qu'elle peut se développer de façons diverses chez différents individus. En effet, bien que les aspects de la connaissance des mots varient d'un auteur à l'autre, les chercheurs s'entendent pour dire que ces aspects se développent de façon relativement indépendante et que l'apprentissage des mots devrait être contextualisé de manière à soutenir le développement de chacun d'eux (Kamil et Hiebert, 2005; Nagy et Scott, 2000; Nonnon, 2012; Schmitt, 2014). Dans la présente étude, l'écriture collaborative situe l'apprentissage des mots cibles dans une activité contextualisée, permettant le développement simultané de ces différentes facettes.

D’un point de vue opérationnel, lorsque le lexique est mesuré dans différentes études, deux dimensions sont considérées : la taille, ou l'étendue, et la profondeur. Il importe donc de se pencher sur ces concepts qui ne sont pas tout à fait séparables. La taille du lexique est une notion qui fait relativement unanimité. Elle fait référence au nombre d'items lexicaux (mots) connus à un certain niveau de maitrise par une personne (Ouellette, 2006; Qian, 2002; Schmitt, 2014). De l'autre côté, la profondeur du lexique est un concept qui suscite un intérêt particulier dans la dernière décennie. Bien qu'il n'existe pas de consensus quant à sa définition (Nonnon, 2012; Qian, 2002; Zhang et Yang, 2016), cet aspect réfère généralement à une connaissance plus ou moins approfondie des aspects des mots (Gablasova, 2014; Kucan, 2012; Ouellette, 2006; Qian, 2002; Schmitt, 2014). Si un parallèle devait être dressé, profondeur et taille du lexique pourraient se rapporter à l'opposition qualité-quantité, la profondeur étant la « qualité » de la connaissance des mots et la taille la « quantité » de mots connus.

Dans le but de mesurer adéquatement la compétence lexicale de nos participants, nous avons pris en compte ces deux dimensions dans le choix des outils de mesure. Ainsi, nous avons décidé d'utiliser deux tests qui mesurent respectivement la taille et la profondeur du vocabulaire pour avoir une idée représentative de la connaissance lexicale de nos participants au début de la recherche. 


\section{Enseignement efficace du lexique et l'écriture collaborative}

Les études sur l'enseignement du lexique, majoritairement en contexte anglophone, ont permis de dégager les principes d'un enseignement systématique et efficace du lexique (Graves, 2006; Beck et coll., 2002). Ces principes invitent entre autres à favoriser des expériences riches et fréquentes avec les mots, l'enseignement de mots individuels selon leur utilité et potentiel d'apprentissage, l'enseignement de stratégies d'apprentissage des mots, telles que se servir des indices du contexte et analyser la structure des mots, et le développement de la conscience des mots.

Ces principes ont inspiré la conception d'outils pédagogiques d'enseignement du lexique, notamment l'utilisation de l'écriture dans l'enseignement du lexique. En effet, une activité d'écriture favorise l'apprentissage actif des nouveaux mots en invitant les élèves à les utiliser dans leur discours et en contexte d'écriture (Ardasheva et coll.., 2019; Beck, McKeown et Kucan, 2008; Graves, 2008; Holec, 2019). Une activité d'écriture semble ainsi idéale afin de travailler le lexique.

Certains auteurs, plus particulièrement en contexte d'anglais langue seconde, ont exploré les effets de l'écriture collaborative sur les apprentissages en milieu scolaire. D'une manière générale, il semble que l'écriture collaborative ait un effet positif sur la compétence à écrire (Shehadeh, 2011). Effectivement, certains auteurs notent que les participants ayant écrit en groupes récoltent de meilleures notes pour leurs travaux écrits et qu'ils développent une vision positive de l'écriture, que ce soit par rapport à leurs capacités en tant qu'écrivains ou par rapport à l'écriture collaborative elle-même (Nassaji et Tian, 2010; Yarrow et Topping, 2001; Shehadeh, 2011). Par ailleurs, des chercheurs soutiennent que les textes écrits par des dyades ou des groupes d'élèves sont plus précis (Dobao, 2012; Storch, 2005). Enfin, différentes études ont rapporté un effet positif de l'écriture collaborative sur différentes composantes de la compétence en écriture, entre autres l'orthographe, la ponctuation, le lexique, l'organisation ou encore le contenu (Shehadeh, 2011; Storch, 2005; Yarrow et Topping, 2001). Ces résultats nous mènent à penser que l'écriture collaborative, ayant un effet positif sur différents apprentissages, pourrait avoir un effet semblable au niveau du lexique. Cependant, on remarque que peu d'études se sont intéressées au lien entre ce type d'écriture et la connaissance des mots.

L'écriture collaborative se définit comme la production d'un texte par deux personnes ou plus travaillant ensemble. Plus spécifiquement, les interactions sociales, la négociation, le conflit cognitif, le partage de connaissances et certains facteurs affectifs facilitants représentent les caractéristiques nécessaires à l'établissement d'un réel travail collaboratif (Fung, 2010). Par ailleurs, l'écriture collaborative se distingue par son processus récursif (Yarrow et Topping, 2001), c'est-à-dire qu'il est possible de passer d'une étape à l'autre de l'écriture (planification, mise en texte, révision) de façon non linéaire. Enfin, le dialogue est un élément clé de l'écriture collaborative, puisqu'il permet aux participants de développer leurs ressources cognitives en se concentrant sur certains aspects de la langue (Kim, 2008). 


\section{Appuis théoriques de la relation entre l'apprentissage du lexique et l'écriture collaborative}

Certaines théories, provenant principalement de la recherche sur le lexique en langue seconde, appuient un effet positif de l'écriture collaborative sur l'apprentissage du lexique. D'abord, la théorie de la production, ou « pushed output » en anglais, suppose que l'utilisation de la langue au travers d'échanges crée des occasions pour les apprenants de se rendre compte des lacunes dans leur utilisation de celle-ci (Bybee, 2008). Dans le cas de l'écriture collaborative, les négociations placeraient l'élève face à ses connaissances et ses lacunes, menant ainsi à une meilleure rétention des mots discutés (Bridgman, 2005).

D'un autre côté, la théorie de la charge d'implication (involvement load), dérivée des sciences cognitives, se base sur le fait qu'un enseignement nécessitant une participation active de l'étudiant aiderait à la construction d'un réseau sémantique plus fort, ce qui mènerait à des apprentissages plus durables (Beck et coll., 2008). Suivant cette théorie, des exercices tels que la création originale de phrases, par exemple lors de l'écriture collaborative, apporteraient un apprentissage plus durable que des exercices à trous, puisque les premiers nécessiteraient un niveau plus profond de traitement de l'information (Parreren, 1995). Certaines études expérimentales ont d'ailleurs trouvé un appui empirique à cette hypothèse (Golonka et coll., 2015; Hulstijn et Laufer, 2001; Kim, 2008).

Enfin, la perspective socioconstructiviste permet, elle aussi, d'expliquer des gains dans l'apprentissage du lexique. Celle-ci postule qu'il est difficile de réaliser un apprentissage de façon individuelle, puisque l'apprentissage est une entreprise sociale en soi (Dobao, 2012; Fung, 2010; Nassaji et Tian, 2010; Shehadeh, 2011). Les interactions en écriture collaborative permettraient ainsi aux étudiants de partager leurs connaissances sur les mots et de bâtir un apprentissage plus solide.

\section{Études empiriques portant sur la relation entre l'écriture collaborative et l'apprentissage du lexique}

À notre connaissance, seules deux études ont exploré spécifiquement les effets de l'écriture collaborative sur les apprentissages. Une première étude de Kim (2008), portant sur la comparaison du niveau d'efficacité de tâches collaboratives et individuelles sur l'acquisition du vocabulaire, a exploré cet effet. Les résultats de cette recherche indiquent que le groupe collaboratif a significativement mieux performé sur les post-tests immédiats et différés au niveau de la rétention des mots que le groupe individuel. À la suite de ces résultats, Kim (2008) a conclu que les étudiants travaillant en collaboration se seraient davantage interrogés sur les mots au travers de leurs interactions que les étudiants individuels réfléchissant à voix haute et auraient ainsi mieux retenu ces mots. Nous avons également répertorié l'étude de Shehadeh (2011) qui a exploré les effets des perceptions de l'écriture collaborative en langue seconde. Les résultats de cette étude ont montré que l'écriture collaborative avait un effet significatif sur l'amélioration de la compétence à écrire et plus spécifiquement sur le sujet, l'organisation et le lexique. Au niveau du lexique, le groupe collaboratif a significativement mieux performé que le groupe individuel sur les aspects de la variation, la précision, la maitrise de la forme du mot, la justesse du registre et l'efficacité dans la transmission du message. 


\section{Synthèse}

Les théories de l'apprentissage collaboratif en langue seconde, tout comme les recherches s'étant penchées sur les effets de l'écriture collaborative sur divers apprentissages, plaident en faveur de son utilisation dans les classes, notamment pour l'apprentissage des mots. Or, peu de recherches ont exploré directement ce lien. Dans ce contexte, notre recherche visait à répondre à la question suivante: «Quel est l'effet d'une séquence d'écriture collaborative sur l'apprentissage du lexique chez des élèves du troisième cycle du primaire scolarisés en français langue première ? »

\section{Méthodologie}

\section{Participants}

Dans le but de répondre à la question de recherche, un échantillon de convenance a été recruté (Fortin et Gagnon, 2016). Deux groupes de $6^{\mathrm{e}}$ année provenant de deux écoles francophones en milieu défavorisé (IMSE 9) de la commission scolaire de Montréal ont participé à la recherche. Le groupe contrôle était composé de 16 participants, soit 6 filles et 10 garçons. Le groupe expérimental était composé de 17 participants, à savoir 6 filles et 11 garçons. Tous les participants étaient âgés de 11 à 12 ans. La majorité (23) des participants parlaient français à la maison, certains parlant également anglais (2), espagnol (3), arabe (2), ou une autre langue (3).

\section{Instruments de mesure}

Pour pouvoir évaluer les effets d'une séquence d'écriture collaborative sur l'apprentissage du lexique, nous avons pris des mesures de l'étendue et de la profondeur du lexique. Pour mesurer l'étendue du lexique des participants, nous avons choisi le test de l'Échelle de vocabulaire en images de Peabody (EVIP), soit une adaptation française du Peabody Picture Vocabulary Test largement utilisée dans les études en littératie. Ce test a été créé pour mesurer l'étendue du vocabulaire réceptif et les normes sont basées sur des élèves francophones canadiens (Dunn, Dunn et Theriault-Whalen, 1993). Les participants devaient choisir une image parmi quatre qui correspondait le mieux à un mot annoncé.

\section{Figure 1}

Exemple du Vocabulary Knowledge Scale adapté

\section{Abattre}

1. Je ne sais pas ce que ce mot signifie

2. J'ai déjà vu ce mot et je pense qu'il veut dire

3. Je connais ce mot, il veut dire (synonyme ou définition)

4. Je peux utiliser ce mot dans une phrase (écris une phrase) 
Pour mesurer la profondeur du lexique, nous avons conçu une nouvelle épreuve à l'instar de l'Échelle de connaissance du vocabulaire (Vocabulary Knowledge Scale de Wesche et Paribakht, 1994). Ce test comportait 15 mots pour lesquels le participant devait entourer l'option qui reflétait le mieux sa connaissance du mot (voir figure 1). Les participants devaient, par ailleurs, fournir une réponse en fonction de l'option choisie. Les points étaient accordés tel que suit, pour un maximum de 5 points par mot ou 75 points au total :
- 0
Le mot n'est pas connu
- 1
Le mot est connu, mais sa définition n'est pas connue
- 2
Une définition ou un synonyme partiel est donné
- 3
Une définition ou un synonyme approprié est donné
- +1
Le mot est utilisé avec un sens approprié dans une phrase
- +2
Le mot est utilisé avec un sens approprié dans une phrase grammaticalement
correcte

\section{Intervention}

Durant l'intervention, le groupe expérimental et le groupe contrôle ont participé à trois séances d'écriture. Ces séances étaient séparées en deux temps, soit l'enseignement des mots cibles et leur transfert dans une situation d'écriture. Lors de la situation d'écriture, les participants du groupe expérimental étaient invités à faire de l'écriture collaborative, c'est-à-dire qu'ils écrivaient un texte en équipes de quatre, tandis que les participants du groupe contrôle écrivaient de façon individuelle.

\section{Enseignement des mots cibles}

Quinze mots cibles ont été choisis pour être enseignés aux deux groupes lors des séances d'écriture. Ce choix a été effectué en suivant le critère d'utilité mentionné par Beck et coll. (2002) et Graves et coll. (2014), soit si le mot est souvent utilisé dans les manuels ou contextes scolaires. Par ailleurs, les mots cibles de l'étude font partie du deuxième Tiers de mots énoncé par Beck et coll. (2002), c'est-à-dire que ce sont des mots dont la fréquence est relativement haute, mais qui permettent de préciser des concepts déjà connus. Les mots de ce Tiers possèdent le meilleur potentiel d'utilité puisqu'ils nécessitent un enseignement, mais qu'ils permettent aussi à l'apprenant d'accéder à un bagage lexical riche. Afin de rendre l'apprentissage des mots cibles pertinent, il était nécessaire de les rendre utiles à la tâche d'écriture. Ainsi, les mots ont été choisis en fonction de trois thèmes, soit l'environnement, l'école et l'alimentation. Au départ, 30 mots cibles respectant les critères mentionnés ci-haut ont été sélectionnés dans Manulex (Lété, Sprenger-Charolles, 2004). La passation du prétest de l'Échelle de connaissance du vocabulaire dans une classe de $6^{\mathrm{e}}$ année nous a permis de valider la cohérence interne des 30 mots cibles. L'analyse des réponses des élèves à l'aide du logiciel SPSS a révélé un alpha de Cronbach très satisfaisant $(\alpha=0,942)$. Dans le but de ne pas créer de surcharge cognitive, nous avons ensuite réduit ce nombre à 15 en suivant les critères suivants : cinq mots par thème choisi; diversifiés dans leur fréquence d'occurrence de façon à ce que nous puissions analyser l'apprentissage des mots en fonction de ce critère; la fiabilité interitem (l'alpha de Cronbach) des 15 mots cibles devait être supérieure à 0,70. Une nouvelle analyse a révélé que les 15 mots cibles choisis en fonction de ces critères avaient une fiabilité interne très 
satisfaisante $(\alpha=0,911)$. Les 15 mots cibles, ainsi que leur fréquence d'occurrence, sont présentés dans le tableau 1.

Tableau 1

Mots cibles et leur fréquence d'occurrence

\begin{tabular}{cccc}
\hline Mot & Fréquence $\mathbf{( U )}$ & Mot & Fréquence $(\mathbf{U})$ \\
\hline Abattre & 10,38 & Mécontent & 8,30 \\
Caprice & 5,80 & Obligé & 23,59 \\
Crampe & 1,01 & Précaution & 21,93 \\
Conservation & 5,31 & Raisonnable & 9,88 \\
Délicieux & 31,31 & Rive & 34,24 \\
Épuisé & 4,80 & Terrible & 21,25 \\
Habile & 30,93 & Touffu & 1,28 \\
Inutile & 3,11 & & \\
\hline
\end{tabular}

Lors de l'intervention, l'enseignement des mots cibles aux deux groupes participants s'est réalisé en trois temps. D'abord, les élèves étaient invités à définir le mot. Ensuite, une chercheuse écrivait la définition du mot au tableau. Enfin, les participants devaient fournir un exemple du mot en contexte dans une phrase. Dans la plupart des cas, les participants avaient déjà une conception du sens du mot présenté et étaient capables de nommer les principales composantes de sa définition. Dans le cas contraire, la chercheuse analysait la structure interne du mot de façon à faire ressortir ses éléments morphologiques. Cela permettait aux élèves de déduire son sens. À la suite de cet enseignement, les participants étaient invités à écrire un texte à l'aide des mots cibles.

\section{Enseignement de la technique d'écriture collaborative}

Afin de nous assurer que les participants du groupe expérimental effectuaient bien de l'écriture collaborative, nous avons créé des équipes pour que les forces et les faiblesses des élèves soient équilibrées, avec l'aide de l'enseignante du groupe. Également, puisqu'il s'agissait d'une expérience relativement nouvelle pour les élèves, nous avons voulu respecter les principes de l'enseignement explicite. Ce type d'enseignement permet de responsabiliser les élèves en réduisant progressivement les groupes de travail (Gauthier, et coll., 2007). Ainsi, une séance a été modélisée par une chercheuse, une séance s'est réalisée en grand groupe et, finalement, trois séances se sont déroulées en équipes de quatre.

Par ailleurs, il a été constaté que les élèves du groupe expérimental avaient déjà écrit en équipe, mais qu'ils n'avaient jamais reçu d'enseignement explicite de l'écriture collaborative. Nous avons donc ajouté une séance d'enseignement explicite concernant la collaboration dans une activité d'écriture en nous basant sur les principes de Speck (2002). Lors de cette séance d'enseignement, une chercheuse a abordé les décisions, les conflits et l'éthique dans un travail d'écriture à plusieurs. Elle a aussi suggéré des stratégies, entre autres la distribution de rôles ou la reformulation de propositions. L'enseignement était suivi d'une modélisation de l'écriture collaborative par une équipe d'élèves volontaires. Les autres participants devaient noter les stratégies utilisées par l'équipe qui favorisaient la collaboration. 


\section{Description des séances}

Les séances se sont déroulées du 12 mars au 2 avril 2019. Elles ont été divisées de façon à ce que chaque groupe participe à deux séances par semaine et que l'apprentissage des mots se réalise simultanément. Toutes les périodes étaient menées par une des chercheuses et se sont déroulées en après-midi. Elles avaient une durée moyenne de 40 minutes.

La première période était divisée en deux temps. La première moitié de la séance consistait à modéliser la technique d'enseignement des mots cibles en enseignant cinq mots tests. Ces mots ne se retrouvaient pas dans l'Échelle de connaissance du vocabulaire. La deuxième partie comprenant une modélisation de l'écriture d'un texte à partir de ces mots tests et d'une image affichée au tableau. La chercheuse écrivait le texte et verbalisait sa démarche d'écriture. Elle engageait graduellement les participants dans l'écriture du texte.

L'enseignement de la technique d'écriture collaborative a été réalisé lors de la deuxième période. Cette période était réservée au groupe expérimental. Le groupe contrôle n'a donc pas suivi de deuxième séance, puisqu'il effectuait de l'écriture individuelle. La troisième séance était divisée en deux parties. La première, d'environ 15 minutes, consistait en l'enseignement de cinq mots cibles liés à l'environnement. La deuxième partie comportait l'écriture d'un texte à partir de ces mots et d'une image, elle aussi liée à l'environnement. Le groupe contrôle écrivait individuellement, tandis que le groupe expérimental écrivait en équipes de quatre élèves. Les séances quatre et cinq se sont déroulées similairement. Pour la quatrième séance, l'image ainsi que les mots cibles étaient liés à la thématique de l'école. Pour la cinquième, ils portaient sur l'alimentation.

\section{Résultats}

Afin de répondre à la question de recherche, nous avons effectué des analyses descriptives, de comparaison de moyennes et de variance (ANOVA). Les résultats des analyses descriptives sont présentés au Tableau 2. Afin de vérifier si les deux groupes étaient comparables au prétest, nous avons effectué un test $t$ à échantillons indépendants sur les résultats au test de l'étendue du vocabulaire, EVIP. L'analyse a révélé que les deux groupes n'étaient pas statistiquement différents $(t=3,162$, ddl $=30, p=0,543)$. Ces résultats suggèrent que les deux groupes étaient égaux au niveau des connaissances lexicales générales. En outre, nous avons voulu vérifier si nos deux groupes différaient statistiquement au niveau de leur connaissance des mots cibles. Un deuxième test $t$ à échantillons indépendants a été effectué à partir des résultats à l'Échelle de connaissance du vocabulaire au prétest, présentés dans le Tableau 2. Ce tableau permet de constater que les deux groupes n'étaient pas statistiquement différents sur la connaissance des mots cibles de l'étude $(t=0,23, \mathrm{ddl}=29, p=0,819)$. 
Tableau 2

Analyses descriptives des résultats des deux groupes à L'EVIP et à l'Échelle de connaissance du vocabulaire

\begin{tabular}{|c|c|c|c|c|c|}
\hline Test & Temps & Groupe & $\mathbf{N}$ & Moyenne & Écart type \\
\hline \multirow[b]{2}{*}{ EVIP } & & Contrôle & 16 & 24.94 & 4.538 \\
\hline & & Expérimental & 16 & 29.75 & 4.058 \\
\hline \multirow{6}{*}{$\begin{array}{c}\text { Échelle de } \\
\text { connaissance du } \\
\text { vocabulaire }\end{array}$} & \multirow[t]{3}{*}{ Prétest } & Contrôle & 15 & 39.31 & 11.464 \\
\hline & & Expérimental & 16 & 40.44 & 8.625 \\
\hline & & Total & 31 & 39.88 & 9.986 \\
\hline & \multirow[t]{3}{*}{ Post-test } & Contrôle & 15 & 52.929 & 15.274 \\
\hline & & Expérimental & 16 & 54.778 & 13.153 \\
\hline & & Total & 31 & 53.969 & 13.913 \\
\hline
\end{tabular}

Pour ce qui est des moyennes à l'Échelle de connaissance du vocabulaire, les résultats montrent que les deux groupes se sont améliorés entre le prétest et le post-test. Par ailleurs, la différence de moyennes entre les deux groupes semble plus grande au post-test qu'au prétest $(1,13$ contre 1,849$)$.

Dans le but de vérifier si le traitement expérimental d'écriture collaborative avait eu un effet sur l'apprentissage du lexique, nous avons effectué une analyse de variance ANOVA à deux facteurs intersujet (groupe expérimental et groupe contrôle) et intrasujets (prétest et post test), à partir des résultats au test de l'Échelle de connaissance du vocabulaire. Les résultats de l'analyse sont présentés dans le tableau 3.

Tableau 3

Analyse de la variance entre les deux groupes

\begin{tabular}{ccccccc}
\hline Source & $\begin{array}{c}\text { Somme des } \\
\text { carrés }\end{array}$ & ddl & $\begin{array}{c}\text { Carré } \\
\text { moyen }\end{array}$ & F & Signification & $\begin{array}{c}\text { Éta-carré } \\
\text { partiel }\end{array}$ \\
\hline Constante & 138934.074 & 1 & 138934.074 & 619.888 & 0 & .955 \\
Groupe & 192.397 & 1 & 192.397 & 0.858 & .362 & .029 \\
Erreur & 6499.7 & 29 & 224.128 & & & \\
\hline
\end{tabular}

L'analyse de variance ANOVA a révélé que la différence entre les deux groupes au post-test n'était pas significative $(F=0,858, p>0,005)$. Cela suggère que les connaissances lexicales sur les mots cibles de notre étude se sont développées également chez les deux groupes. Pour mieux comprendre nos résultats, nous avons poursuivi notre analyse en calculant le nombre de mots obtenus pour chaque score pour chacun des groupes. Les résultats de cette analyse sont présentés dans le tableau 4. 
Tableau 4

Nombre d'items lexicaux pour chaque notation par groupe

\begin{tabular}{ccccccccc}
\hline & $N$ & Nb total de mots & \multicolumn{5}{c}{ Nombre de mots par notation } \\
\hline $\begin{array}{c}\text { Notation } \\
\text { Prétest }\end{array}$ & & 0 & 1 & 2 & 3 & 4 & 5 \\
Contrôle & 16 & 240 & 59 & 22 & 31 & 27 & 41 & 60 \\
Expérimental & 16 & 240 & 31 & 41 & 24 & 58 & 46 & 40 \\
Post-test & & & & & & & & \\
Contrôle & 15 & 225 & 25 & 13 & 26 & 37 & 37 & 87 \\
Expérimental & 17 & 225 & 9 & 20 & 22 & 37 & 42 & 125 \\
\hline
\end{tabular}

Chaque participant dans chaque groupe a rencontré 15 mots cibles dans l'Échelle de connaissance du vocabulaire. Ainsi, pour chaque groupe, le total de mots testés était le nombre de participants multiplié par le nombre de mots cibles (15). En ce qui concerne le groupe contrôle, ce tableau nous permet de constater que les connaissances des participants lors du prétest se trouvaient dans les extrêmes, exprimées par un plus grand nombre de notations 0 et 5 . Pour le groupe expérimental, c'est la notation 3 qui était la plus souvent obtenue lors du prétest, indiquant que les participants connaissaient la définition des mots. Au niveau du post-test, c'est le score 5 qui a été obtenu le plus souvent chez les deux groupes. En outre, il faut noter que le groupe expérimental a plus que doublé le nombre de mots connus au plus haut score, contrairement à ce qui a été observé chez le groupe contrôle.

Enfin, nous avons souhaité vérifier s'il existait un lien entre la fréquence d'usage des mots et l'apprentissage de ceux-ci. Le tableau 5 présente les résultats totaux obtenus par chaque mot lors du prétest et du post-test. Le résultat total est obtenu en additionnant le score de chaque élève $(0,1,2,3,4$ ou 5$)$ d'un même groupe pour un mot ciblé.

Tableau 5

Résultat par mot pour chaque groupe

\begin{tabular}{lcccc}
\hline Mots & $\begin{array}{c}\text { Proupe } \\
\text { Contrôle }^{\mathrm{a}}\end{array}$ & $\begin{array}{c}\text { Groupe } \\
\text { expérimental }^{\mathrm{a}}\end{array}$ & $\begin{array}{c}\text { Groupe } \\
\text { contrôle }^{\mathrm{b}}\end{array}$ & $\begin{array}{c}\text { Post-test } \\
\text { expérimental }^{\mathrm{c}}\end{array}$ \\
\hline Abattre & 40 & 48 & 56 & 60 \\
Caprice & 17 & 25 & 29 & 29 \\
Conservation & 28 & 36 & 37 & 41 \\
Crampe & 35 & 50 & 39 & 53 \\
Délicieux & 68 & 61 & 56 & 75 \\
Épuisé & 65 & 61 & 50 & 71 \\
Habile & 46 & 44 & 51 & 67 \\
Inutile & 65 & 62 & 67 & 75 \\
Mécontent & 40 & 53 & 54 & 82 \\
Obligé & 62 & 61 & 61 & 81 \\
Précaution & 39 & 40 & 49 & 65 \\
Raisonnable & 30 & 23 & 47 & 64 \\
Rive & 21 & 21 & 46 & 76 \\
Terrible & 51 & 41 & 58 & 75 \\
Touffu & 22 & 21 & 59 & \\
\hline
\end{tabular}

Note. ${ }^{\mathrm{a}}$ Maximum de 80 points ; ${ }^{\mathrm{b}}$ Maximum de 75 points ; ${ }^{\mathrm{c}}$ Maximum de 85 points 
Un test de corrélation de Pearson a montré qu'il n'existait pas de relation significative entre la fréquence d'usage d'un mot et la connaissance que nos participants avaient de ce mot, que ce soit au prétest $(r=0,126, p=0,656)$ ou au post-test $(r=0,257, p$ $=0,355)$. Ces résultats suggèrent que la fréquence d'usage des mots n'a pas affecté l'apprentissage de nos participants.

\section{Discussion}

La présente recherche visait à déterminer l'effet d'une séquence d'écriture collaborative sur l'apprentissage du lexique chez des élèves de $6^{\mathrm{e}}$ année. Les résultats des analyses indiquent qu'il n'y a pas de différence significative au niveau de l'apprentissage des mots entre les deux groupes après leur séquence d'écriture collaborative ou d'écriture individuelle respective $(\mathrm{F}=0.858, \mathrm{p}>0.005)$. Nos résultats ne corroborent pas les données d'études similaires à la nôtre. En effet, les recherches de Kim (2008) et Shehadeh (2011) suggéraient une amélioration significative du lexique à la suite d'activités d'écriture collaborative chez les élèves du groupe collaboratif comparés à ceux qui écrivaient individuellement. Or, il faut noter que ces études ont été réalisées en contexte d'anglais langue seconde, alors que notre recherche abordait un contexte de français langue première. En ce sens, il est possible que les apprenants de langue seconde bénéficient davantage des activités d'écriture collaborative pour l'apprentissage du lexique que leurs homologues en langue première.

Afin de comprendre plus encore la divergence de nos résultats par rapport aux études mentionnées ci-haut, nous avons effectué une analyse plus détaillée des résultats. Nous avons observé que l'écart entre les deux groupes semblait se creuser entre le prétest et le post-test, bien que la différence des moyennes ne fût pas significative. Cet effet est constaté à partir des résultats présentés dans le tableau 4, où le groupe expérimental a plus que doublé le nombre de mots ayant reçu le plus haut score entre le prétest et le post-test, représentant une amélioration de $212.5 \%$. Quant au groupe contrôle, ce nombre n'a augmenté que de 27 points, soit une hausse de $45 \%$. Considérant que notre analyse ne comporte que deux temps, il est donc possible que la durée de l'expérimentation ne fût pas assez longue pour que nous puissions voir des changements significatifs dans les apprentissages.

En outre, il faut mentionner que nos résultats corroborent les données d'autres chercheurs. En effet, Storch (2005) rapportait dans son étude qu'il n'y avait pas de différence significative entre les textes produits par des dyades et ceux produits par des élèves individuels, bien que les dyades aient mieux performé à priori. Même si ces conclusions ne concernent pas l'apprentissage du lexique, nous observons un parallèle entre ces résultats et nos données. Nos résultats suivent également ceux de Nassaji et Tian (2010). Ces auteurs avaient trouvé que les participants s'étaient significativement améliorés dans leurs connaissances des verbes à l'étude, mais qu'il n'y avait pas de différences significatives entre la condition collaborative et la condition individuelle. Puisque nous avons également observé une amélioration chez les participants entre le prétest et le posttest, mais que la différence entre les deux groupes n'était pas significative, il est concevable que le contexte d'écriture soit un facteur d'apprentissage plus important que la collaboration sur la tâche. Il serait intéressant d'explorer davantage cette voie en comparant 
les effets d'activités collaboratives d'écriture, de lecture ou orales sur l'apprentissage du lexique.

Finalement, pour mieux comprendre nos résultats, nous avons aussi voulu vérifier s'il existait une relation entre la fréquence d'usage des mots et leur apprentissage. Les résultats de notre analyse de corrélation indiquent qu'il n'existe pas de relation significative entre la fréquence d'un mot et sa maitrise par nos participants au prétest et au post-test. Ces résultats suggèrent que les mots moins fréquents sont appris au même rythme que leurs homologues plus fréquents.

\section{Limites de la recherche}

Une limite majeure de la présente recherche est la taille de l'échantillon. En effet, la puissance statistique de nos analyses a été limitée par le nombre réduit de participants et la grande variation dans leurs résultats, ce qui pourrait expliquer l'absence d'effet de la séquence d'écriture collaborative sur l'apprentissage du lexique. De toute évidence, de futures recherches sont nécessaires pour vérifier cette hypothèse. En ce qui a trait au traitement utilisé dans notre étude, celui-ci pourrait être bonifié. Puisque plusieurs auteurs conseillent d'enseigner les mots en contexte (Beck et coll., 2002; Gablasova, 2015; Nagy et Scott, 2000; Schmitt, 2014), l'ajout d'un court texte où se seraient retrouvés les mots cibles enseignés aurait pu favoriser leur apprentissage. De la même façon, le nombre limité de séances a pu contribuer au manque de puissance statistique de nos analyses. Un dispositif expérimental contenant un nombre plus élevé de séances aurait probablement davantage effet sur les apprentissages des participants. Enfin, notre Échelle de connaissance du vocabulaire, adaptée du Vocabulary Knowledge Scale (Wesche et Paribakht, 1994), est un test de rappel. Puisque les participants devaient produire une réponse, il est possible que ce test ait manqué de sensibilité pour capter les premiers apprentissages lexicaux. Un test de reconnaissance, par exemple un questionnaire à choix multiples, aurait pu être davantage adapté pour mesurer les nouveaux apprentissages.

\section{Implications pour la recherche et le milieu scolaire}

À notre connaissance, notre étude est la première à s'intéresser à l'effet de l'écriture collaborative sur le lexique en milieu francophone. Bien qu'ils soient non concluants, les résultats de notre recherche ouvrent la porte à d'autres études de plus grande envergure qui exploreraient les effets de l'écriture collaborative comme dispositif d'enseignement du lexique. Par ailleurs, notre adaptation du Vocabulary Knowledge Scale (Wesche et Paribakht, 1994) contribue à la réflexion menée sur la mesure de la profondeur du lexique, puisque ce concept retient l'attention des chercheurs dans les écrits récents. Enfin, bien que nos résultats non significatifs ne nous permettent pas de suggérer l'écriture collaborative comme une piste prometteuse de l'enrichissement du lexique chez les élèves, l'amélioration des résultats des élèves du groupe expérimental invite les chercheurs à vérifier ses effets en tenant compte des limites de notre étude. Il a effectivement été démontré au travers de la problématique que les connaissances lexicales sont intimement liées à la réussite scolaire des élèves (Ardasheva et coll., 2019; Binder et coll., 2017; Zhang et Yang, 2016) et qu'il existe un réel manque de temps associé à leur enseignement (Anctil, 2011; Beck, Mckeown et Kucan, 2002; Scott, Jamieson-Noel et Asselin, 2003). Conséquemment, davantage 
d'études portant sur des méthodes d'enseignement du lexique éprouvées par la recherche doivent être réalisées.

La correspondance devrait être adressée à Maude Roy-Vallières.

Courriel :mauderoyv@hotmail.com

\section{Références}

Anctil, D. (2011). L'erreur lexicale au secondaire : analyse d'erreurs lexicales d'élèves de 3e secondaire et description du rapport à l'erreur lexicale d'enseignants de français (Thèse de doctorat). Université de Montréal. https://papyrus.bib.umontreal.ca/xmlui/handle/1866/5077

Ardasheva, Y., Hao, T. et Zhang, X. (2019). Research-driven pedagogy. Routledge.

Beacco, J. C., Fleming, M., Goullier, F., Thürmann, E., et Vollmer, H. (2016). Les dimensions linguistiques de toutes les matières scolaires: Guide pour l'élaboration des curriculums et pour la formation des enseignants. Conseil de l'Europe.

Beck, I. L., Mckeown, M. G. et Kucan, L. (2002). Bringing words to life : robust vocabulary instruction. Solving problems in the teaching of literacy. Guilford Press.

Beck, I. L., McKeown, M. G. et Kucan, L. (2008). Creating robust vocabulary : Frequently asked questions and extended examples, vol.10. Guilford Press.

Binder, K. S., Cote, N. G., Lee, C., Bessette, E. et Vu, H. (2017). Beyond breadth : the contributions of vocabulary depth to reading comprehension among skilled readers. Journal of research in reading, 40(3), 333-343.

Bolger, D. J., Balass, M., Landen, E. et Perfetti, C. A. (2008). Context variation and definitions in learning the meanings of words : An instance-based learning approach. Discourse processes, 45(2), 122-159.

Bridgman, B. (2005). Vocabulary acquisition in the communicative classroom. Rapport annuel du Centre International de l'Université Ryukoku, (14), 121-128.

Bybee, J. (2008). Usage-based grammar and second language acquisition. Dans Handbook of cognitive linguistics and second language acquisition, pp. 226-246. Routledge.

Dobao, A. F. (2012). Collaborative writing tasks in the 12 classroom : Comparing group, pair, and individual work. Journal of Second Language Writing, 21(1), 40-58.

Dreyfus, M. (2004). Pratiques et représentations de l'enseignement du lexique à l'école primaire. Dans 9 e colloque de l'Association Internationale pour la Recherche en Didactique du Français.

Dunn, L. M., Dunn, L. M. et Theriault-Whalen, C. (1993). EVIP : échelle de vocabulaire en images Peabody. Pearson Canada Assessment.

Fortin, F. et Gagnon, J. (2016). Fondements et étapes du processus de recherche: méthodes quantitatives et qualitatives (3e édition.). Chenelière éducation.

Fung, Y. M. (2010). Collaborative writing features. RELC Journal, 41(1), 18-30.

Gablasova, D. (2014). Learning and retaining specialized vocabulary from textbook reading : Comparison of learning outcomes through 11 and 12. The Modern Language Journal, 98(4), 976-991.

Gablasova, D. (2015). Learning technical words through 11 and 12 : Completeness and accuracy of word meanings. English for Specific Purposes, 39, 62-74.

Gauthier, C., Bissonnette, S. et Richard, M. (2007). L’enseignement explicite. 
Golonka, E., Bowles, A., Silbert, N., Kramasz, D., Blake, C. et Buckwalter, T. (2015). The role of context and cognitive effort in vocabulary learning : A study of intermediate-level learners of arabic. The Modern Language Journal, 99(1), 19-39.

Graves, M. F. (2006). The vocabulary book : learning \& instruction. Language and literacy series. Teacher's College Press.

Graves, M. F. (2008). Teaching individual words : One size does not fit all. New York : Teachers College Press.

Graves, M. F., Baumann, J. F., Blachowicz, C. L., Manyak, P., Bates, A., Cieply, C., Davis, J. R. et Von Gunten, H. (2014). Words, words everywhere, but which ones do we teach? The Reading Teacher, 67(5), 333-346.

Guthrie, J. T., Wigfield, A., Barbosa, P., Perencevich, K. C., Taboada, A., Davis, M. H., Scafiddi, N. T. et Tonks, S. (2004). Increasing reading comprehension and engagement through concept-oriented reading instruction. Journal of educational psychology, 96(3), 403-423.

Holec, H. (2019). Structures lexicales et enseignement du vocabulaire : thèse de IIIème cycle (Vol. 5). De Gruyter GmbH \& Co KG.

Hulstijn, J. H. et Laufer, B. (2001). Some empirical evidence for the involvement load hypothesis in vocabulary acquisition. Language learning, 51(3), 539-558.

Kamil, M. L. et Hiebert, E. H. (2005). Teaching and learning vocabulary. Dans E. H. Hiebert et M.L. Kamil (dir.), Teaching and learning vocabulary: Bringing research to practice tice, (Vol.1, pp.1-26). Lawrence Erlbaum Associates Inc.

Kim, Y. (2008). The contribution of collaborative and individual tasks to the acquisition of 12 vocabulary. The Modern Language Journal, 92(1), 114-130.

Kucan, L. (2012). What is most important to know about vocabulary? The Reading Teacher, 65(6), 360-366.

Kwary, D. A. (2011). A hybrid method for determining technical vocabulary. System, 39(2), 175-185.

Larousse (2018). Dictionnaires de français larousse. Récupéré de $\mathrm{http}$ ://www.larousse.fr/dictionnaires/francais-monolingue

Lété, B., Sprenger-Charolles, L. et Colé, P. (2004). Manulex : A grade-level lexical database from french elementary school readers. Behavior Research Methods, Instruments, \& Computers, 36(1), 156-166.

Magnusson, C. G., Roe, A., et Blikstad-Balas, M. (2019). To what extent and how are reading comprehension strategies part of language arts instruction? A study of lower secondary classrooms. Reading Research Quarterly, 54(2), 187-212.

Nagy, W. E. et Scott, J. A. (2000). Vocabulary processes. Dans M. L. Kamil, P. B. Mosenthal, P. D. Pearson et R. Barr (dir.), Handbook of reading research (vol.3, p.269-284). Lawrence Erlbaum Associates Inc.

Nassaji, H. et Tian, J. (2010). Collaborative and individual output tasks and their effects on learning english phrasal verbs. Language Teaching Research, 14(4), 397-419.

Nation, I. (1990). Teaching and Learning Vocabulary. Dans E. Hinkel (Eds.), Handbook of Research in Second Language Teaching and Learning (p.581-596). Lawrence Erlbaum Associates Inc. https://books.google.ca/books?id=IQd\_QgAACAAJ

Nonnon, É. (2012). La didactique du français et l'enseignement du vocabulaire, dans vingt ans de revues de didactique du français langue première. Repères. Recherches en didactique du français langue maternelle, (46), 33-72. 
Ouellette, G. P. (2006). What's meaning got to do with it: The role of vocabulary in word reading and reading comprehension. Journal of educational psychology, 98(3), 554.

Oxford, R. L. (1997). Cooperative learning, collaborative learning, and interaction : Three communicative strands in the language classroom. The Modern Language Journal, 81(4), 443-456.

Paradis, M. (2009). Declarative and procedural determinants of second languages (Studies in bilinguism, 40). Amsterdam: John Benjamins.

Parreren, C. S.-v. (1995). Action psychology as applied to foreign language vocabulary acquisition. Computer Assisted Language Learning, 8(2-3), 181-204.

Perez Bedoya, S. (2018). L'acquisition de la graphomotricité chez les élèves francophones au début du primaire (Mémoire de maitrise). Université de Sherbrooke.

Picoche, J. (2011). Lexique et vocabulaire : quelques principes d'enseignement à l'école. Récupéré de http://cache.media.eduscol.education.fr/file/Dossier_vocabulaire/14/4/Jacqueline_Pi coche_111202_avec_couv_201144.pdf

Qian, D. D. (2002). Investigating the relationship between vocabulary knowledge and academic reading performance : An assessment perspective. Language learning, 52(3), 513-536

Schmitt, N. (2014). Size and depth of vocabulary knowledge : What the research shows. Language Learning, 64(4), 913-951

Scott, J. A., Jamieson-Noel, D. et Asselin, M. (2003). Vocabulary instruction throughout the day in twenty-three canadian upper-elementary classrooms. The Elementary School Journal, 103(3), 269-286.

Shehadeh, A. (2011). Effects and student perceptions of collaborative writing in 12. Journal of Second Language Writing, 20(4), 286-305.

Speck, B. W. (2002). Facilitating Students' Collaborative Writing. ASHE-ERIC Higher Education Report. Jossey-Bass Higher and Adult Education Series. ERIC.

Stahl, S. A. (2005). Four problems with teaching word meanings. Teaching and learning vocabulary : Bringing research to practice, 95-114.

Storch, N. (2005). Collaborative writing : Product, process, and students' reflections. Journal of second language writing, 14(3), 153-173.

Swain, M. (2001). Integrating language and content teaching through collaborative tasks. Canadian Modern Language Review, 58(1), 44-63.

Tolmie, A. K., Topping, K. J., Christie, D., Donaldson, C., Howe, C., Jessiman, E., Livingston, K. et Thurston, A. (2010). Social effects of collaborative learning in primary schools. Learning and instruction, 20(3), 177-191.

Vinterek, M., Winberg, M. T., Tegmark, M., Alatalo, T., et Liberg, C. (2018). Amount of text read at school and the motivation for reading: A large scale study in grade 6 and 9. Dans European Conference on Educational Research, Bolzano, Italy, September 4-7, 2018.

Wesche, M. et Paribakht, T. S. (1994). Enhancing vocabulary acquisition through reading $\therefore$ A hierarchy of text-related exercise types. Communication présentée à la AAAL '94 Conference, Baltimore, Maryland.

Yarrow, F. et Topping, K. J. (2001). Collaborative writing: The effects of metacognitive prompting and structured peer interaction. British journal of educational psychology, 71(2), 261-282. 
Yonek, L. M. (2009). The effects of rich vocabulary instruction on students' expository writing. (Thèse de doctorat). University of Pittsburgh.

Zhang, D. et Yang, X. (2016). Chinese 12 learners' depth of vocabulary knowledge and its role in reading comprehension. Foreign Language Annals, 49(4), 699-715. 